\title{
THE RELATIONS BETWEEN SPHERICAL, CYLINDRICAL, AND SPHEROIDAT HARMONICS
}

\author{
$B y$ G. B. JEFFERY.
}

[Received and Read May 11th, 1916.-Revised September 16th, 1916.]

Is a paper published in the Mathematische Annalen* in 1903, Whittaker has shown that a general solution of Laplace's equation may be written in the form

$$
\int_{0}^{2 \pi} f(x \cos t+y \sin t+i z, t) d t .
$$

In the same paper he gave the integrals of this type corresponding to spherical and cylindrical harmonies. With the usual notation

$r^{n} P_{n}^{m}(\cos \theta){ }_{\sin }^{\cos } m \phi=\frac{i^{m-n}}{2 \pi} \frac{\Gamma(n+m+1)}{\Gamma(n+1)} \int_{0}^{2 \pi}(x \cos t+y \sin t+i z)^{n}{ }_{\sin }^{\cos } m t d t,(1)$ and

$e^{-k z} J_{m}(k \pi) \underset{\sin }{\cos } m \phi=\frac{i^{-m}}{2 \pi} \int_{0}^{2 \pi} e^{i l_{k}(x \cos t+y \sin t+i z)} \frac{\cos }{\sin } m t d t$.

More recently E. Blades $\dagger$ has shown that interior spheroidal harmonics are given by

$P_{n}^{m}(i \xi) P_{n}^{m}(\cos \eta) \underset{\sin }{\cos } m \phi=\frac{1}{2 \pi} \frac{(n+m) !}{(n-m) !} \int_{0}^{2 \pi} P_{n}\left(\frac{x \cos t+y \sin t+i z}{c}\right){ }_{\sin }^{\cos } m t d t$,

where the coordinates are defined by

$$
x=c \sqrt{\hat{\xi}^{2}+1} \sin \eta \cos \phi, \quad y=c \sqrt{\hat{\xi}^{2}+1} \sin \eta \sin \phi, \quad z=c \xi \cos \eta,
$$

while the author has shown + that the corresponding harmonics which vanish at infinity are given by

$$
Q_{n}^{m}(i \xi) P_{n}^{n n}(\cos \eta) \sin _{\sin }^{\cos } m \phi=\frac{1}{2 \pi} \frac{(n+m) !}{(n-m) !} \int_{0}^{2 \pi} Q_{n}\left(\frac{x \cos t+y \sin t+i z}{c}\right){ }_{\sin }^{\cos } m t d t
$$

* Vol. Lvir, p. 333.

$\dagger$ Proc. Edinburgh Mathematical Society, Vol. xxxirr, 1915.

$\ddagger$ Ibid. 
Without examining the extreme range of the values of the variables for which the formulæ (1), (2), (3), (5), are valid, it will be sufficient for our purpose to note that they all hold good if $z>|c|$.

The object of the present paper is to show that Whittaker's general solution provides a ready means of expressing a given potential function in terms of different harmonics. Thus if we can expand the function $f$ in a series of powers of its first argument, we can express the potential in spherical harmonics; if we expand in a Fourier series we have the same potential expressed in terms of Bessel functions; while if we desire to use spheroidal harmonics we must expand $f$ in a series of Legendre functions.

We shall find it convenient to write

$$
x \cos t+y \sin t+i z \equiv \xi .
$$

To transform spherical to spheroidal harmonics, having the same axis and origin.-Let $c$ be the parameter of the spheroidal coordinates. If $c$ is pure imaginary the coordinate surfaces $\xi=$ const. are confocal oblate spheroids; if $c$ is real they are problate spheroids having a common focal circle of radius $|c|$. We will confine our investigation to spherical harmonics of integral degree. Those of positive degree are included in the set

$$
r^{n} P_{n}^{n n}(\cos \theta) \cos m \phi=\frac{i^{n-3 n}}{2 \pi} \frac{(n+m) !}{n !} \int_{0}^{2 \pi} \xi^{n} \cos m t d t,
$$

it being understood that throughout the paper $\cos m \phi$ may be replaced,by $\sin m \phi$, if at the same time cos $m t$ is replaced by sin $m t$. By a well known result (Ferrers, Spherical Harmonics, p. 27)

$$
\left(\frac{\zeta}{c}\right)^{n}=n ! \sum_{p=0}^{q} \frac{(2 n-4 p+1)(n-p) !}{(2 n-2 p+1) ! p !} 2^{n-2 p} P_{n-2 p}\left(\frac{\xi}{c}\right),
$$

where $q$ is $\frac{1}{2} n$ or $\frac{1}{2}(n-1)$, whichever is an integer.

Using this result and afterwards substituting from (3), we have $r^{n} P_{n}^{m}(\cos \theta) \cos m \phi$

$$
\begin{aligned}
= & \frac{i^{m-n}}{2 \pi} \frac{(n+m) !}{n !} \int_{0}^{2 \pi} \xi^{n} \cos m t d t \\
= & \frac{c^{n}}{2 \pi i^{i n-n}}(n+m) ! \int_{0}^{2 \pi} \sum_{p=0}^{q} \frac{(2 n-4 p+1)(n-p) !}{(2 n-2 p+1) ! p !} 2^{n-2_{p}} P_{n-2_{p}}\left(\frac{\xi}{c}\right) \cos m t d t \\
= & \frac{c^{n}}{i^{n-m}}(n+m) ! \cos m \phi \\
& \quad \times \sum_{p=0}^{q} \frac{(2 n-4 p+1)(n-p) !(n-2 p-m) !}{(2 n-2 p+1) !(n-2 p+m) ! p !} 2^{n-2_{p} p} P_{n-2_{2} p^{n}}^{n}(i \xi) P_{n-2_{p}}^{n t}(\cos \eta) .
\end{aligned}
$$


If $n$ is a negative integer, let it be $-\nu-1$; then, $m$ being a positive integer,

$$
\frac{\Gamma(n+m+1)}{\Gamma(n+1)}=\frac{\sin (\nu+1) \pi}{\sin (\nu-m+1) \pi} \frac{\Gamma(\nu+1)}{\Gamma(\nu-m+1)}=(-1)^{m} \frac{\nu !}{(\nu-m) !},
$$

and (1) may be written

$$
\frac{1}{r^{\nu+1}} P_{\nu}^{m}(\cos \theta) \cos m \phi=\frac{i^{\nu-m+1}}{2 \pi} \frac{\nu !}{(\nu-m) !} \int_{0}^{2 \pi} \xi^{-\nu-1} \cos m t d t .
$$

Now $\xi^{-\nu-1}$ regarded as a function of the complex variable $\xi$ is holomorphic at all points outside any ellipse $C$ whose foci are the points \pm 1 . It may therefore be expanded by Neumann's theorem in a series of Legendre functions of the second kind. It may be shown that if $\xi$ lies outside an ellipse whose foci are $\pm c$,

$$
\xi^{-\nu-1}=\frac{1}{c^{\nu+1} \nu !} \sum_{p=0}^{\infty}(-1)^{p} \frac{(2 \nu+4 p+1)(2 \nu+2 p) !}{2^{\nu+2 p}(\nu+p) ! p !} Q_{\nu+2 p}\left(\frac{\xi}{c}\right) .
$$

The spherical harmonics which vanish at infinity are therefore given by $\frac{1}{r^{\nu+1}} P_{\nu}^{m}(\cos \theta) \cos m \phi$

$=\frac{i^{\nu-m+1}}{2 \pi c^{\nu+1}} \frac{1}{(\nu-m) !} \int_{0}^{2 \pi} \sum_{p=0}^{\infty}(-1)^{p} \frac{(2 \nu+4 p+1)(2 \nu+2 p) !}{2^{\nu+2 p}(\nu+p) ! p !} Q_{\nu+2 p}\left(\frac{\xi}{c}\right) \cos m t d t$

$=\frac{i^{\nu-m+1}}{c(\nu-m) !} \cos m \phi$

$\times \sum_{p=0}^{\infty}(-1)^{p} \frac{(2 \nu+4 p+1)(2 \nu+2 p) !(\nu+2 p-m) !}{2^{\nu+2 p}(\nu+p) !(\nu+2 p+m) ! p !} Q_{\nu+2 p}^{m}(i \xi) P_{\nu+2 p}^{m}(\cos \eta)$,

by equation (5).

In arriving at this result we have assumed that $\xi$ lies outside any ellipse of a certain family. This is equivalent to the assumption that $\xi$ is not a real quantity between $\pm|c|$. It is readily seen that this condition is satisfied if $z>|c|$, whether $c$ be real or imaginary. Now the series on the right-hand side of (8) converges uniformly if $i \xi$ is finitely different from unity, and allowing for the fact that $\xi, \eta$ are not at all points continuous functions of $\varpi, z$, its space gradient is continuous if $\xi \neq 0$. In the case of both oblate and problate spheroids these conditions are satisfied outside any spheroid of the system $\xi=$ const. In this region both sides of (8) are solutions of Laplace's equation, which, with their first differential coefficients, are finite and continuous. They have been proved equal when $z>|c|$; hence (8) is true everywhere outside any ellipse $\xi=$ const. 
To transform spherical to cylindrical harmonics, having the same axis and origin.-From Hankel's contour integral for the $\Gamma$-function we readily obtain

$$
\Gamma(n+1) \xi^{-n-1}=i^{-n-1} \int_{0}^{\infty} e^{i k \zeta} k^{n} d k,
$$

provided that $n+1>0$ and the real part of $i \xi$ is negative, i.e., $z>0$.

Hence

$$
\begin{aligned}
\frac{1}{r^{n+1}} P_{n}^{m}(\cos \theta) \cos m \phi & =\frac{i^{n-m+1}}{2 \pi} \frac{n !}{(n-m) !} \int_{0}^{2 \pi} \xi^{-n-1} \cos m t d t \\
& =\frac{i^{-m}}{2 \pi(n-n) !} \int_{0}^{2 \pi} \int_{0}^{\infty} e^{i k \zeta} k^{n} \cos m t d k d t \\
& =\frac{\cos m \phi}{(n-m) !} \int_{0}^{\infty} e^{-k z} J_{n}(k \varpi) k^{n} d k,
\end{aligned}
$$

by (2), if $z>0$.

This result was given by Hobson.* It expresses any spherical harmonic which vanishes at infinity in terms of Bessel functions. Hobson also gave a contour integral, involving Bessel functions of complex argument, for the spherical harmonics which are finite at the origin. This may be obtained in the same way.

To transform cylindrical to spherical harmonics. - Expanding the exponential in (2) in powers of its argument and then using (1), we have

$$
\begin{aligned}
e^{-k z} J_{m}(k \varpi) \cos m \phi & =\frac{i^{-m}}{2 \pi} \int_{0}^{2 \pi} e^{i k \zeta} \cos m t d t \\
& =\frac{i^{-m}}{2 \pi} \int_{0}^{2 \pi} \sum_{p=0}^{\infty} \frac{(i k)^{p}}{p !} \xi^{p} \cos m t d t \\
& =\cos m \phi \sum_{p=0}^{\infty} \frac{(-1)^{m+p}}{(m+p) !} k^{p} r^{p} P_{p}^{m}(\cos \theta),
\end{aligned}
$$

or, changing the sign of $k$,

$$
e^{k z} J_{m}(k \varpi) \cos m \phi=\cos m \phi \sum_{p=0}^{\infty} \frac{(k r)^{p}}{(m+p) !} P_{p}^{m}(\cos \theta) .
$$

The result was also given by Hobson in the paper referred to above. It has been proved for $z>|c|$, but, both sides of the equation (11) being

* "Bessel's Funotions and Relations connecting them with Spherical Harmonics," Proc. Loindon Math. Soc., 1893, Vol, xxv, p. 73. 
continuous potential functions everywhere in the finite region, this restriction may be removed.

To transform cylindrical to spheroidal harmonics, having the same origin and axes.-Bauer* has given the expansion

$$
e^{i k \zeta}=\left(\frac{2 \pi}{c k}\right)^{\frac{1}{2}} \sum_{n=0}^{\infty}(2 n+1) i^{n} J_{n+\frac{1}{2}}(c k) P_{n}\left(\frac{\zeta}{c}\right) .
$$

By Neumann's theorem on the expansion of a function in a series of Legendre functions, the expansion is valid for all finite values of $\zeta$, for $e^{i k \zeta}$ is holomorphic for all finite values of $\xi$. From this result, together with (2) and (3), we have

$$
\begin{aligned}
& e^{-k z} J_{m}(k \varpi) \cos m \phi \\
& \quad=\left(\frac{2 \pi}{c k}\right)^{\frac{1}{2}} \cos m \phi \sum_{n=0}^{\infty} i^{n-m}(2 n+1) \frac{(n-m) !}{(n+m) !} J_{n+\frac{1}{2}}(c k) P_{n}^{m}(i \xi) P_{n}^{m}(\cos \eta) .
\end{aligned}
$$

This result is valid for all finite values of $\xi$.

To transform spheroidal to spherical harmonics, having the same axes and origin.-First consider the internal spheroidal harmonics. We have

$$
P_{n}^{m}(i \xi) P_{n}^{m}(\cos \eta) \cos m \phi=\frac{1}{2 \pi} \frac{(n+m) !}{(n-m) !} \int_{0}^{2 \pi} P_{n}\left(\frac{\xi}{c}\right) \cos m t d t .
$$

Now

$$
P_{n}\left(\frac{\xi}{c}\right)=\sum_{p=0}^{q}(-1)^{p} \frac{(2 n-p) !}{2^{n} p !(n-p) !(n-2 p) !}\left(\frac{\xi}{c}\right)^{n-2 p},
$$

where $q=\frac{1}{2} n$ or $\frac{1}{2}(n-1)$, whichever is an integer. The corresponding transformations when $n$ is not an integer, but the value of $\xi$ is suitably restricted, can be obtained by the same method if they are required. From the above results and (1) we have

$P_{n}^{m}(i \hat{\xi}) P_{n}^{m}(\cos \eta) \cos m \phi$

$=\frac{i^{n-m}}{2^{n}} \frac{(n+m) !}{(n-m) !} \cos m \phi \sum_{p=0}^{q} \frac{(2 n-p) !}{(n-p) !(n+m-2 p) ! p !}\left(\frac{r}{c}\right)^{n-2 p} P_{n-2 p}^{n}(\cos \theta)$.

The external harmonics are given by

$$
Q_{n}^{m}(i \xi) P_{n}^{m}(\cos \eta) \cos m \phi=\frac{1}{2 \pi} \frac{(n+m) !}{(n-m) !} \int_{0}^{2 \pi} Q_{n}\left(\frac{\xi}{c}\right) \cos m t d t,
$$


and

$$
Q_{n}\left(\frac{\xi}{c}\right)=2^{n} \sum_{p=0}^{\infty} \frac{(n+p) !(n+2 p) !}{(2 n+2 p+1) ! p !}\left(\frac{c}{\xi}\right)^{n+2 p+1},
$$

provided that

$$
|x \cos t+y \sin t+i z|>|c|
$$

for all real values of $t$, i.e. that

$$
z>|c| \text {. }
$$

Hence, using (7),

$Q_{n}^{m}(i \xi) P_{n}^{m}(\cos \eta) \cos m \phi$

$$
\begin{aligned}
& =\frac{2^{n-1}}{\pi} \frac{(n+m) !}{(n-m) !} \int_{0}^{2 \pi} \sum_{p=0}^{\infty} \frac{(n+p) !(n+2 p) !}{(2 n+2 p+1) ! p !}\left(\frac{c}{\xi}\right)^{n+2 p+1} \cos m t d t \\
& =\frac{2^{n}}{i^{n-m+1}} \frac{(n+m) !}{(n-m) !} \cos m \phi \sum_{p=0}^{\infty} \frac{(n+p) !(n-m+2 p) !}{(2 n+2 p+1) ! p !}\left(\frac{c}{r}\right)^{n+2 p+1} P_{n+2 p}^{m}(\cos \theta) .
\end{aligned}
$$

By the same argument as we have used above, (13) is true everywhere, and (14) is true outside the sphere $r=|c|$.

To transform spheroidal to cylindrical harmonics.-If $z>|c|$, we have

$$
Q_{n}\left(\frac{\xi}{c}\right)=2^{n} \sum_{p=0}^{\infty} \frac{(n+p) !(n+2 p) !}{(2 n+2 p+1) ! p !}\left(\frac{c}{\xi}\right)^{n+2 p+1},
$$

and from (9) in the same circumstances

$$
\frac{1}{\xi^{n+2 p+1}}=\frac{(-1)^{p}}{i^{n+1}(n+2 p) !} \int_{0}^{\infty} e^{i k \zeta} k^{n+2 p} d k .
$$

Hence $\quad Q_{n}\left(\frac{\xi}{c}\right)=\frac{2^{n} c}{i^{n+1}} \sum_{p=0}^{\infty} \frac{(-1)^{p}(n+p) !}{(2 n+2 p+1) ! p !} \int_{0}^{\infty} e^{i k \zeta(c k)^{n+2 p}} d k$.

We may interchange the order of the summation and integration and, after reducing the factorials, we have

$$
Q_{n}\left(\frac{\xi}{c}\right)=\frac{c \Gamma\left(\frac{1}{2}\right)}{2 i^{i+1}} \int_{0}^{\infty} e^{i k \zeta} \sum_{p=0}^{\infty} \frac{(-1)^{p}}{p ! \Gamma\left(n+p+\frac{3}{2}\right)}\left(\frac{c k}{2}\right)^{n+2 p} d k
$$

which, by the series for the Bessel functions, gives

$$
Q_{n}\left(\frac{\xi}{c}\right)=i^{-n-1} \int_{0}^{\infty}\left(\frac{c \pi}{2 k}\right)^{\frac{1}{2}} e^{i k \zeta} J_{n+\frac{1}{2}}(c k) d k .
$$


Hence, using (2) and (5), we have without further difficulty $Q_{n}^{m}(i \xi) P_{n}^{m}(\cos \eta) \cos m \phi$

$$
=i^{m-n-1} \frac{(n+m) !}{(n-m) !} \cos m \phi \int_{0}^{\infty}\left(\frac{c \pi}{2 k}\right)^{\frac{1}{3}} J_{n+\frac{1}{2}}(c k) e^{-k z} J_{m}(k \pi) d k,
$$

if $z>0$.

Whittaker's integral may also be used to obtain relations between harmonics of the same kind referred to different axes. No great generality can be expected in this direction, as the integral itself does not admit some very simple changes of axes. In some cases, however, the formulæ of transformation are very readily obtained. Amongst those we may mention Bessel's formulæ for changing the origin of spherical harmonics whilst retaining the same axis, and Nicholson's formule connecting Bessel harmonics referred to parallel axes.

In this paper we have confined our attention to three important types of harmonies, but the same method may be used to investigate the relations between these and ellipsoidal, paraboloidal, toroidal, bipolar, and elliptic and parabolic cylinder harmonics, all of which may be expressed in Whittaker's form. 